\title{
The strength of the atom localization coupling with the standing-wave field under the dephasing rate influence
}

\author{
S. Abd-El-Nabi \\ Department of Mathematics, Faculty of Science (Women's Section) P. O. Box 11754, Al-Azhar University, Nasr City, \\ Cairo, Egypt.
}

Accepted 29 March, 2013

\begin{abstract}
We investigate the localization of the four-level V-type atom inside a classical standing-wave field through the absorption spectrum of the weak probe field. The expression of the probe absorption spectrum is derived analytically via perturbation treatment of a weak probe field. Numerical results and discussion of the probe susceptibility spectrum behaviour via non-perturbation treatment of the density matrix elements to all orders of fields are obtained. We have shown that the precision of localization of the atom is dependent on the dephasing rates of atomic coherence as well as on some parameters of the driving and standing-wave fields. A strong localization through sharply peak is achieved at a certain value for the dephasing rates in resonance detunings.
\end{abstract}

Key words: Quantum optics, atomic system, atom localization.

\section{INTRODUCTION}

Many different techniques to measure the position of the atom passing through a standing-wave field have developed in recent years. The interaction of a singlemode quantized standing-wave cavity field with a threelevel atom passing through the Ramsey-type experimental have been studied in (Kien et al., 1997). Several schemes have been considered for the localization of the atom using the standing optical light. One of the simple scheme has studied the atom localization inside the standing-wave field during its motion based on resonance fluorescence (Qamer et al., 2000) and $Q$ distribution function through a Kerr-like medium (Osman, 2005). The precision of the position measurement of the atom depends upon the relative Phase of the driving fields, whereas the phase of the standing-wave driving field has an important role in reducing the number of localization peaks from the usual four to two, leading to a new localization scheme which is called sub-half-wavelength localization, this is demonstrated in Ghafoor et al. (2002) and Sahrai et al. (2005). Atomic coherence effect, such as coherent population trapping have been shown to be useful for sub-wavelength localization of the atom by Agarwal and Kapale (2006). The sub-wavelength localization of an ensemble of atoms via super fluorescence was investigated by Macovei et al. (2007). Jun et al. (2008) discussed the sub-half-wavelength localization of a two level atom via tri-chromatic phase manipulation. Ding et al. (2011) showed that the localization is significantly improved due to the interference effect between the spontaneous decay channels and the dynamically induced quantum interference generated by the two standing-wave fields. The one- and two-dimensional atom localization behaviors via spontaneous emission in a coherently driven five-level atomic system by means of a radio-frequency field driving a hyperfine transition are investigated by Wang et al. (2012). It is found that the detecting probability and precision of $1 \mathrm{D}$ and $2 \mathrm{D}$ atom 


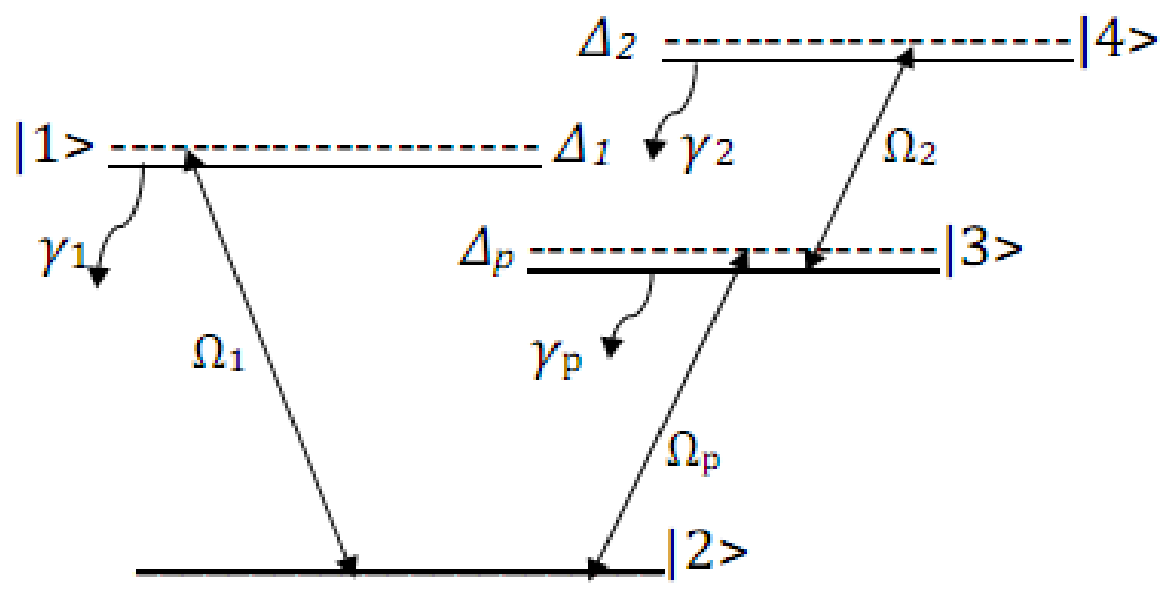

Figure 1. The scheme of a four-level V-type atom interacting with the weak probe and two driving fields.

localization behaviors can be significantly improved via adjusting the system parameters. In this paper we consider a closed four-level atom based on V-type system to determine the position of an atom inside the classical standing-wave field in the presence of the dephasing rate of atomic coherence. The dephasing rate can be preserved in semiconductor double quantum wells (SDQW). It is originated from electron- electron scattering and electron-phonon scattering or even from elastic interface roughness scattering. The semiconductor double quantum wells are very promising candidate in coming years to have a practical multilevel system with the dephasing rate. This is because of controllability in design parameters of such systems (Faist et al., 1997). The tunneling through the barrier can give rise dephasing rate in SDQW (Affolderbach et al., 2002). So we have shown that the strength of the interaction of the atom with the standing-wave field depends on the dephasing rate of atomic coherence. To ascertain the localization, we will determine the absorption of the weak probe field and analyze analytical and numerical results through the imaginary part of the susceptibility. Next, we will investigate the effects of the detuning parameters on the precision of localizing an atom. Finally the conclusion is presented.

\section{MODEL AND EQUATIONS}

We consider a closed four-level atom based on V-type configuration as shown in Figure 1. A weak probe field of frequency (amplitude) $\omega_{\mathbf{p}}\left(\mathrm{E}_{\mathbf{p}}\right)$ is applied to the transition $|2>\leftrightarrow| 3>$ with a Rabi frequency $\Omega_{\mathbf{p}}=\mathrm{E}_{\mathbf{p}} \mathrm{d}_{32} / \hbar$, where $\mathrm{d}_{32}$ is the electric dipole moment. The Hamiltonian of the system in the rotating-wave approximation (Scully and Zubairy, 1997) can be written as:

$\mathrm{H}=\mathrm{H}_{0}+\mathrm{H}_{1}$ where,

$$
\begin{aligned}
& \mathrm{H}_{\mathrm{o}}=-\hbar \Delta_{1} \sigma_{11}-\hbar \Delta_{\mathrm{p}} \sigma_{33}-\hbar\left(\Delta_{\mathrm{p}}+\Delta_{2}\right) \sigma_{44} \\
& \mathrm{H}_{\mathrm{I}}=-\frac{1}{2} \hbar\left(\Omega_{1} \sigma_{12}+\Omega_{\mathrm{p}} \sigma_{32}+\Omega_{2} \sigma_{43}+\text { H.C. }\right)
\end{aligned}
$$

$\omega_{i}$ and $\Omega_{i}(i=1,2)$ are the frequencies and the Rabi frequencies for the coupling and pumping fields which are applied to the transitions $|1>\leftrightarrow| 2>$ and $|3>\leftrightarrow| 4>$ respectively. The detunings are denoted by $\Delta_{1}=\omega_{1} \omega_{12}, \Delta_{p_{2}} \omega_{p_{-}} \omega_{32}$ and $\Delta_{2}=\omega_{2} \omega_{43}$, where $\omega_{12}, \omega_{32}$ and $\omega_{43}$ represent the atomic transition frequencies. $\sigma_{i j}{ }^{i}=|i><j|(i, j=1,2,3,4)$ are the projection operators for $\mathrm{i}=\mathrm{j}$ and the dipole operators for $\mathrm{i} \neq \mathrm{j}$.

The master equation for the density matrix (Meystre and Sergent, 1999) to our model takes the following form:

$\frac{\partial \rho}{\partial t}=-\frac{i}{\hbar}[H, \rho]+\gamma_{1} \mathcal{J}_{21} \rho+\gamma_{p} \mathcal{J}_{23} \rho+\gamma_{2} \mathcal{J}_{34} \rho$

Where,

$\mathcal{J}_{\mathrm{ij}} \rho=\frac{1}{2}\left(2 \sigma_{\mathrm{ij}} \rho \sigma_{\mathrm{ji}}-\sigma_{\mathrm{ji}} \sigma_{\mathrm{ij}} \rho-\rho \sigma_{\mathrm{ji}} \sigma_{\mathrm{ij}}\right)$

The spontaneous decay rates from the atomic levels $|4>\rightarrow| 3>$, $|3>\rightarrow| 2>$ and $\mid 1>$ to the ground level $\mid 2>$ are labeled as $\gamma_{2}, Y_{p}$ and $Y_{1}$ respectively. According to Equation (4), we can obtain the equations of motion for the density matrix elements as (for simplicity, we take $\hbar=1$ and the Rabi frequencies are real):

$$
\frac{\partial \rho_{11}}{\partial t}=-\gamma_{1} \rho_{11}-\frac{i}{2} \Omega_{1}\left(\rho_{12}-\rho_{21}\right)
$$

$\frac{\partial \rho_{33}}{\partial t}=-\gamma_{p} \rho_{33}+\gamma_{2} \rho_{44}-\frac{i}{2} \Omega_{2}\left(\rho_{34}-\rho_{43}\right)+\frac{i}{2} \Omega_{p}\left(\rho_{23}-\rho_{32}\right)$ 


$$
\begin{aligned}
& \frac{\partial \rho_{44}}{\partial \mathrm{t}}=-\gamma_{2} \rho_{44}+\frac{\mathrm{i}}{2} \Omega_{2}\left(\rho_{34}-\rho_{43}\right) \\
& \frac{\partial \rho_{12}}{\partial \mathrm{t}}=\left[\mathrm{i} \Delta_{1}-\frac{1}{2}\left(\gamma_{1}+\Gamma_{12}\right)\right] \rho_{12}-\frac{\mathrm{i}}{2} \Omega_{\mathrm{p}} \rho_{13}+\frac{\mathrm{i}}{2} \Omega_{1}\left(\rho_{22}-\rho_{11}\right)
\end{aligned}
$$

$$
\frac{\partial \rho_{31}}{\partial \mathrm{t}}=\left[-\mathrm{i}\left(\Delta_{1}-\Delta_{\mathrm{p}}\right)-\frac{1}{2}\left(\gamma_{1}+\gamma_{\mathrm{p}}+\Gamma_{31}\right)\right] \rho_{31}-\frac{\mathrm{i}}{2} \Omega_{1} \rho_{32}+\frac{\mathrm{i}}{2} \Omega_{2} \rho_{41}+\frac{\mathrm{i}}{2} \Omega_{\mathrm{p}} \rho_{21}
$$

$$
\frac{\partial \rho_{32}}{\partial \mathrm{t}}=\left[\mathrm{i} \Delta_{\mathrm{p}}-\frac{1}{2}\left(\gamma_{\mathrm{p}}+\Gamma_{32}\right)\right] \rho_{32}-\frac{\mathrm{i}}{2} \Omega_{1} \rho_{31}+\frac{\mathrm{i}}{2} \Omega_{2} \rho_{42}+\frac{\mathrm{i}}{2} \Omega_{\mathrm{p}}\left(\rho_{22}-\rho_{33}\right)
$$

$$
\frac{\partial \rho_{42}}{\partial \mathrm{t}}=\left[\mathrm{i}\left(\Delta_{2}+\Delta_{\mathrm{p}}\right)-\frac{1}{2}\left(\gamma_{2}+\Gamma_{42}\right)\right] \rho_{42}-\frac{\mathrm{i}}{2} \Omega_{1} \rho_{41}+\frac{\mathrm{i}}{2} \Omega_{2} \rho_{32}-\frac{\mathrm{i}}{2} \Omega_{\mathrm{p}} \rho_{43}
$$

$$
\frac{\partial \rho_{41}}{\partial \mathrm{t}}=\left[-\mathrm{i}\left(\Delta_{1}-\Delta_{2}-\Delta_{\mathrm{p}}\right)-\frac{1}{2}\left(\gamma_{1}+\gamma_{2}+\Gamma_{41}\right)\right] \rho_{41}-\frac{\mathrm{i}}{2} \Omega_{1} \rho_{42}+\frac{\mathrm{i}}{2} \Omega_{2} \rho_{31}
$$

$$
\frac{\partial \rho_{43}}{\partial \mathrm{t}}=\left[\mathrm{i} \Delta_{2}-\frac{1}{2}\left(\gamma_{2}+\gamma_{\mathrm{p}}+\Gamma_{43}\right)\right] \rho_{43}-\frac{\mathrm{i}}{2} \Omega_{\mathrm{p}} \rho_{42}+\frac{\mathrm{i}}{2} \Omega_{2}\left(\rho_{33}-\rho_{44}\right)
$$

$\Gamma_{i \bar{j}}$ denotes the dephasing rate of atomic coherence $\boldsymbol{P}_{\mathrm{ij}}(i \neq j)$. We have the trace condition:

$$
\rho_{11}+\rho_{22}+\rho_{33}+\rho_{44}=1 \quad \text { and } \quad \rho_{\mathrm{ij}}=\rho_{\mathrm{ij}}^{*}
$$

Considering the atom moving in z-direction and passing through a classical standing -wave field aligned along the $x$-direction. We assume that the center- of-mass position of the atom is nearly constant along the direction of the standing - wave. Hence, we apply the Raman-Nath approximation (Meystre and Sergent, 1999) and neglect the kinetic part of the atom from the Hamiltonian and the corresponding term from the density matrix elements. The demonstration of the localization atom is achieved when the driving field with Rabi frequency is replaced by a standing-wave field in the above equations. So, we assume the classical standing-wave field is coupled the transition $|3>\leftrightarrow| 4>$ with the frequency $\omega_{2}$, thus the Rabi frequency $\Omega_{2}$ is replaced in Equations 6 to 14, by $\Omega_{2(\mathrm{x})=} \Omega_{2}$ sinkx, where $\mathrm{k}$ is the wave number of the classical standing-wave field with the amplitude $\Omega_{2}$. The first -order solution for the off- diagonal density matrix $\rho_{32}$ (which measures the absorption of the probe field) can be obtained in the steady-state by solving Equations 6 to 14. Perturbatively, for a weak probe field (for simplicity, we assume

$\gamma_{1}=\gamma_{\mathrm{p}}=\gamma_{2}=\gamma, \Gamma_{\mathrm{ij}}=\Gamma_{0}=0$, for $(\mathrm{i} \neq \mathrm{j})$ and $\left.\Delta_{1}=\Delta_{2}=\Delta\right)$,

thus we conclude:

$\rho_{32}^{(1)}=-2 i \Omega_{p}\left[\frac{4\left[i\left(\Delta+\Delta_{p}\right)-1 / 2 \gamma\right]\left[-i\left(\Delta-\Delta_{p}\right)-\gamma\right]\left[i \Delta_{p}-\gamma\right]+\left[-i\left(\Delta-\Delta_{p}\right)-\gamma\right] \Omega_{1}^{2}+\left[i\left(\Delta+\Delta_{p}\right)-1 / 2 \gamma\right]\left(\Omega_{2} \operatorname{sinkx}\right)^{2}}{A_{2}+2 B_{2}\left(\Omega_{2} \sin k x\right)^{2}+\left(\Omega_{2} \sin k x\right)^{4}}\right]$

where,

$$
\begin{aligned}
& A_{2}=16\left[i\left(\Delta+\Delta_{\mathrm{p}}\right)-1 / 2 \gamma\right]\left[\mathrm{i} \Delta_{\mathrm{p}}-1 / 2 \gamma\right]\left[-\mathrm{i}\left(\Delta-\Delta_{\mathrm{p}}\right)-\gamma\right]\left[\mathrm{i} \Delta_{\mathrm{p}}-\gamma\right] \\
& \quad+\Omega_{1}^{2}\left[\Omega_{1}^{2}+4\left[-\mathrm{i}\left(\Delta-\Delta_{\mathrm{p}}\right)-\gamma\right]\left[\mathrm{i} \Delta_{\mathrm{p}}-1 / 2 \gamma\right]+4\left[i\left(\Delta+\Delta_{\mathrm{p}}\right)-1 / 2 \gamma\right]\left[\mathrm{i} \Delta_{\mathrm{p}}-\gamma\right]\right] \\
& \mathrm{B}_{2}=2\left(\left[-\mathrm{i}\left(\Delta-\Delta_{\mathrm{p}}\right)-\gamma\right]\left[\mathrm{i} \Delta_{\mathrm{p}}-\gamma\right]+\left[\mathrm{i}\left(\Delta+\Delta_{\mathrm{p}}\right)-1 / 2 \gamma\right]\left[\mathrm{i} \Delta_{\mathrm{p}}-1 / 2 \gamma\right]\right)-\Omega_{1}^{2}
\end{aligned}
$$

So, the expression for the absorption of probe field can be represented as $\operatorname{Im}\left(\rho_{32}^{(1)}\right)$, according to Equation (16):

$$
\operatorname{Im}\left(\rho_{32}^{(1)}\right)=2 \Omega_{p} \frac{\left(y_{2} k_{2}-x_{2} n_{2}\right)}{\left(k_{2}^{2}+n_{2}^{2}\right)}
$$

Where,

$$
\begin{aligned}
\mathrm{x}_{2}= & \left(\Delta+\Delta_{\mathrm{p}}\right)\left(\Omega_{2} \operatorname{sinkx}\right)^{2}-\left(\Delta-\Delta_{\mathrm{p}}\right) \Omega_{1}^{2}+4 \mathrm{~m}_{1}\left(\Delta+\Delta_{\mathrm{p}}\right)-2 \gamma^{2}\left(\Delta-2 \Delta_{\mathrm{p}}\right), \\
\mathrm{y}_{2}= & 1 / 2 \mathrm{\gamma}\left(\Omega_{2} \operatorname{sinkx}\right)^{2}+\gamma^{2} \Omega_{1}^{2}+\mathrm{\gamma} \mathrm{m}_{1}+4 \mathrm{\gamma}\left(\Delta+\Delta_{\mathrm{p}}\right)\left(\Delta-2 \Delta_{\mathrm{p}}\right), \\
\mathrm{k}_{2}= & \left(\Omega_{2} \operatorname{sinkx}\right)^{4}+\left(\Omega_{2} \operatorname{sinkx}\right)^{2}\left[4\left(\mathrm{~m}_{1}+\mathrm{l}_{1}\right)-2 \Omega_{1}^{2}\right]+\Omega_{1}^{4}+4\left(\gamma^{2}-2 \Delta_{\mathrm{p}}^{2}\right) \Omega_{1}^{2} \\
& \quad+8\left[2 \mathrm{~m}_{1} \mathrm{l}_{1}+\gamma^{2}\left(\Delta^{2}-4 \Delta_{\mathrm{p}}^{2}\right)\right], \\
\mathrm{n}_{2}= & 2 \mathrm{\gamma}\left(\Delta-6 \Delta_{\mathrm{p}}\right)\left(\Omega_{2} \operatorname{sinkx}\right)^{2}-2 \mathrm{\gamma}\left(\Delta+6 \Delta_{\mathrm{p}}\right) \Omega_{1}^{2}+8 \mathrm{\gamma}\left[2\left(\Delta-2 \Delta_{\mathrm{p}}\right) \mathrm{l}_{1}-\left(\Delta+2 \Delta_{\mathrm{p}}\right) \mathrm{m}_{1}\right],
\end{aligned}
$$

The $\operatorname{Im}\left(\rho_{32}^{(1)}\right)$ have peaks at:

$$
\Delta_{\mathrm{p}}= \pm 1 / 2\left[\sqrt{\Delta^{2}+\Omega_{1}^{2}} \pm \sqrt{\Delta^{2}+\left(\Omega_{2} \sin \mathrm{kx}\right)^{2}}\right]
$$

and they are located at the positions:

$$
\mathrm{kx}= \pm \sin ^{-1}\left\{\frac{1}{\Omega_{2}} \sqrt{4 \Delta_{\mathrm{p}}^{2}+\Omega_{1}^{2} \pm 4 \Delta_{\mathrm{p}} \sqrt{\Omega_{1}^{2}+\Delta^{2}}}\right\}+\mathrm{n} \pi
$$

The width (W) of any peak in the absorption spectrum characterizing the degree of localization is given by:

$$
W=\left|\begin{array}{l}
\sin ^{-1}\left\{\frac{1}{\Omega_{2}} \sqrt{4\left(\Delta_{\mathrm{p}}+\frac{\gamma}{2}\right)^{2}+\Omega_{1}^{2} \pm 4\left(\Delta_{\mathrm{p}}+\frac{\gamma}{2}\right) \sqrt{\Omega_{1}^{2}+\left(\Delta+\frac{\gamma}{2}\right)^{2}}}\right\}- \\
\sin ^{-1}\left\{\frac{1}{\Omega_{2}} \sqrt{4\left(\Delta_{\mathrm{p}}-\frac{\gamma}{2}\right)^{2}+\Omega_{1}^{2} \pm 4\left(\Delta_{\mathrm{p}}-\frac{\gamma}{2}\right) \sqrt{\Omega_{1}^{2}+\left(\Delta-\frac{\gamma}{2}\right)^{2}}}\right\}
\end{array}\right|
$$

It is noted that the width decreases with increase in amplitude of the position-dependent on Rabi frequency $\Omega_{2}$ and the localization of the atom depends on the detuning of the standing-wave coupling field (compare to that found for three-level model in Paspalakis and Knight, 2001). In the next section, we present numerical results for the probe absorption spectrum to propose the high resolution localization via the probe absorption measurement.

\section{NUMERICAL RESULTS AND DISCUSSION}

We performed the numerical calculations based on the exact solution of the Equations 6 to 14, under the steadystate condition $\frac{\partial P_{\text {iij }}}{\partial \mathrm{t}}=0$, to obtain the probe field absorption $\rho_{32}$. Here we are interested in the probe absorption 


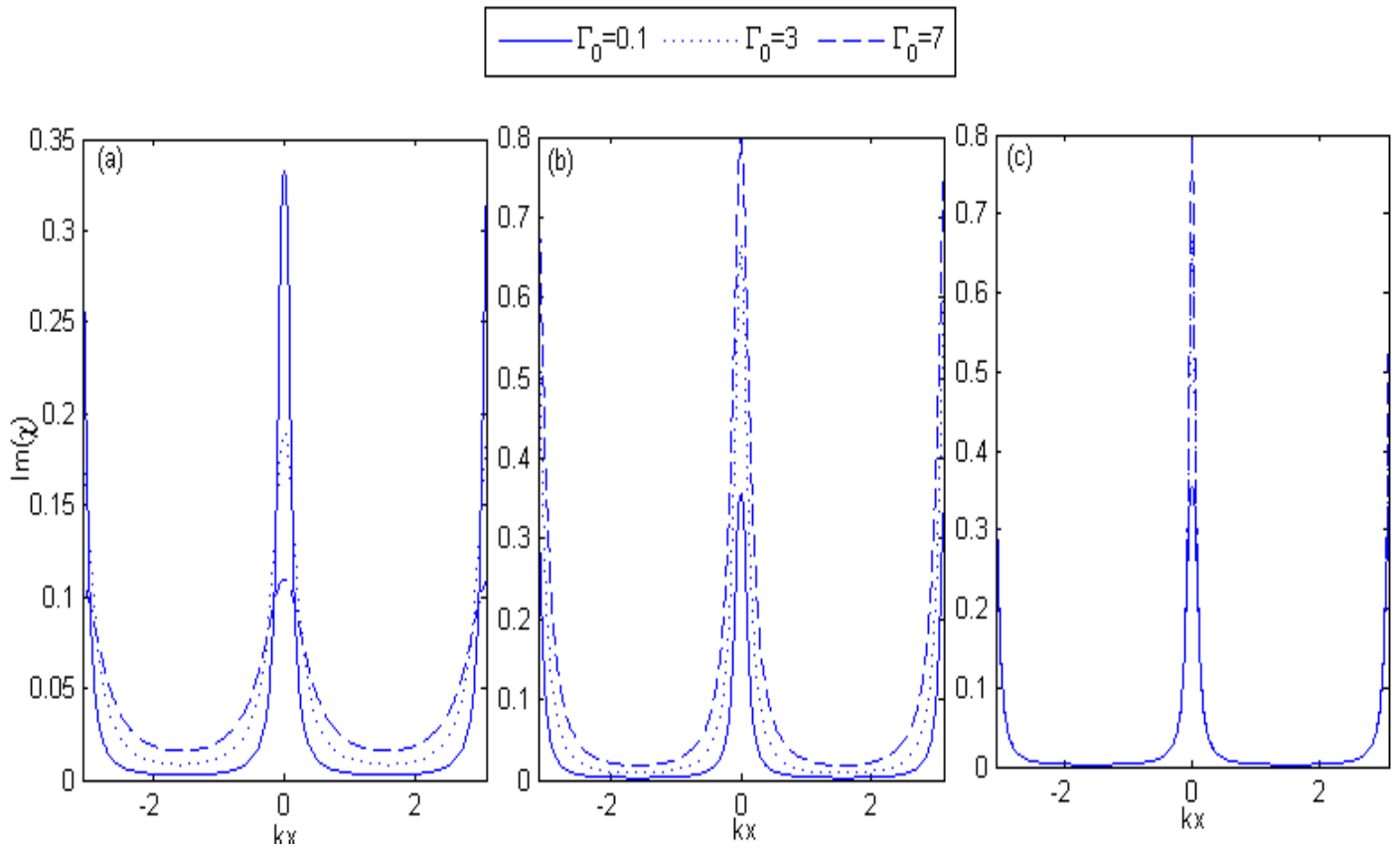

Figure 2. The $2 \mathrm{D}$ absorption spectrum of $\operatorname{Im}(\mathrm{x})$ as a function of $k x(-\pi k x \leq \pi)$ for $\gamma_{1}=Y_{2}=Y_{p}=\gamma$, $\Omega_{1}=\mathrm{y}, \Omega_{2}=20 \mathrm{y}, \Delta_{\mathrm{p}}=\Delta_{2}=\Delta_{1}=0$, (a) $\Gamma_{\mathrm{ij}}=\Gamma_{0}=0.1 \mathrm{\gamma}, 3 \mathrm{y}, 7 \mathrm{\gamma}$, (b) $\Gamma_{\mathrm{ij}}^{\mathrm{v}}$ as in (a) except $\Gamma_{23}=0$, (c) $\Gamma_{\mathrm{ij}}^{\mathrm{s}}$ as in (a) except $\Gamma_{23}=\Gamma_{24}=0$.

spectrum, which is given by the imaginary part of the susceptibility $X=\frac{2 \mathrm{Nd}_{2 s} P_{s 2}}{s_{0} \mathrm{E}_{\mathrm{p}}}$, where $\mathrm{N}$ is the atomic density. Note that the imaginary part of the susceptibility depends on the position $x$ through the term sinkx, thus the absorption spectrum of the weak probe field carries the information on the position along the standing-wave field. For simplicity, it is assumed that the relaxation rates are the same for all levels, that is, $\gamma_{1}=\gamma_{2}=\gamma_{p}=\gamma$, all quantities are scaled in the unit of $\mathrm{Y}$ and the absorption spectra are in the unit of $\left(\frac{2 \mathrm{~N}] \mathrm{d}_{2 \mathrm{I}} I^{2}}{\mathrm{~s}_{\mathrm{o}} \hbar}\right)$.

In Figure 2, we take the 2D absorption spectrum of $\operatorname{Im}(\mathrm{X})$ as a function of $\mathrm{kx}(\pi \leq \mathrm{kx} \leq \pi)$. We choose $\Omega_{\mathrm{p}}=0.001 \mathrm{\gamma}, \Omega_{1}=\gamma$ and $\Omega_{2}=20 \mathrm{\gamma}$, and the detuning

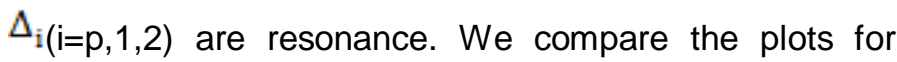
several values of the dephasing rates $\Gamma_{\mathrm{ij}}=\Gamma_{\mathrm{o}}=0.1 \mathrm{y}, 3 \mathrm{\gamma}$, $7 \mathrm{y}$, in Figure $2 \mathrm{a}, \mathrm{b}, \mathrm{c}$. In Figure $2 \mathrm{a}$ we take all the dephase rates have the same values above, and show that when increasing the dephasing rate the height of the peak in the absorption spectra decreases. Figure $2 b$ as in

Figure $2 \mathrm{a}$ but we have $\Gamma_{23}=0$, and show that when increasing the dephasing rate the height and width of the peak in the absorption spectra increases until reach approximately fixed value $(\operatorname{Im}(X)=1)$ whatever the value of the dephasing rate. Figure $2 \mathrm{c}$ as in Figure $2 \mathrm{a}$ but $\Gamma_{23}=\Gamma_{24}=0$, and show that a little high in the peak with increasing the dephasing rate with sharply peak until reach approximately fixed value $(\operatorname{Im}(X)=1)$ whatever the value of the dephasing rate also. Thus, strong localization of the atom can occur.

In Figure 3 , as the same in Figure 2 but we take the off resonance condition $\Delta_{\mathrm{i}}=4 \quad(\mathrm{i}=\mathrm{p}, 1,2)$. It shows that four peaks of the absorption spectra which are decreasing with increasing the dephasing rate. A simple change in the peaks with increasing the dephasing rate are shown in Figure $3 \mathrm{c}$ as well as the sub-wavelength localization of an atom can be achieved.

Figures 4 to 6 are presented as a three-dimensional plots of the absorption spectra $\operatorname{Im}(X)$ versus $k x(-\pi \leq k x$ $\leq \pi)$ for various values of one of the detuning $\Delta_{\mathrm{i}}(\mathrm{i}=\mathrm{p}, 1,2)$, while the other two detuning are equally for both resonance and off- resonance at fixed values $(0,4 \gamma)$ respectively. We take the parameters of the field as in Figure 2.

Figure 4 shows the 3D absorption profiles of $\operatorname{Im}(X)$ as a function of $k x(-\pi \leq k x \leq \pi)$ with various probe detuning $\Delta_{p}$ for both the detunings $\Delta_{1}$ and $\Delta_{2}$ are equal to zero (resonance) or nonzero (off resonance) and $\Gamma_{0}=3 y, \quad \Gamma_{23}=\Gamma_{24}=0$. For resonance condition 


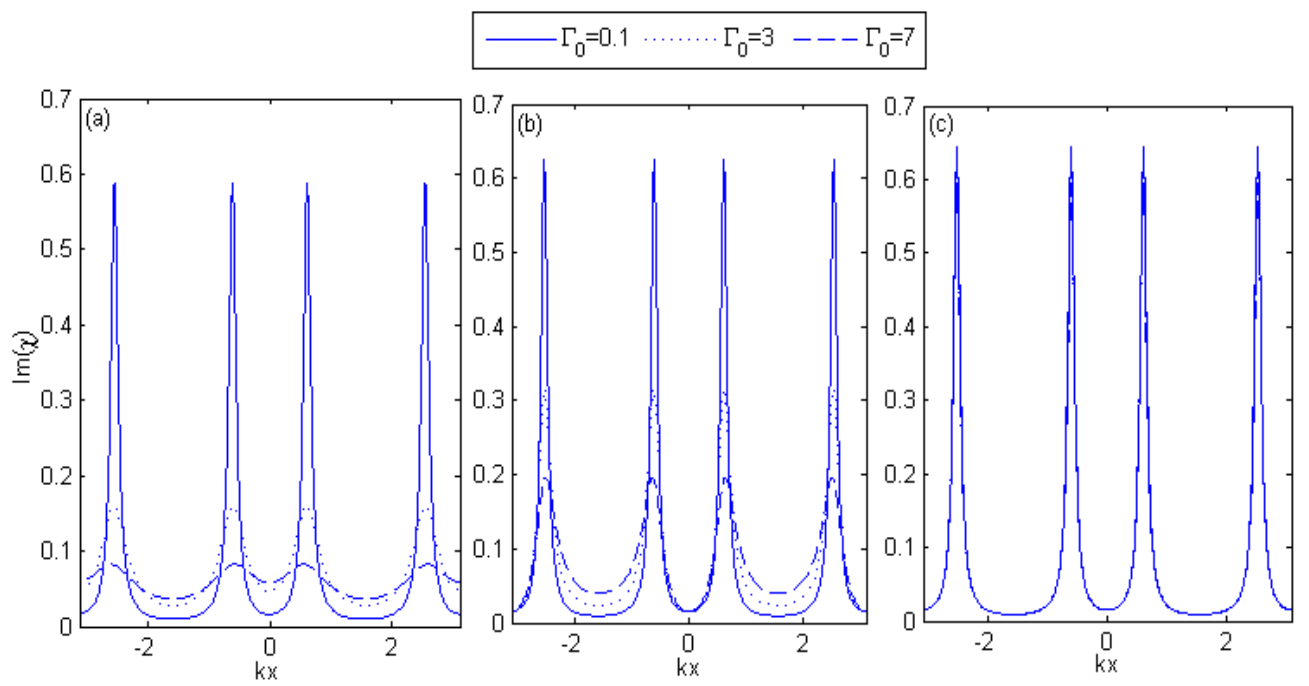

Figure 3. As the same in Figure 2 but we have $\Delta_{p}=\Delta_{2}=\Delta_{1}=4$.
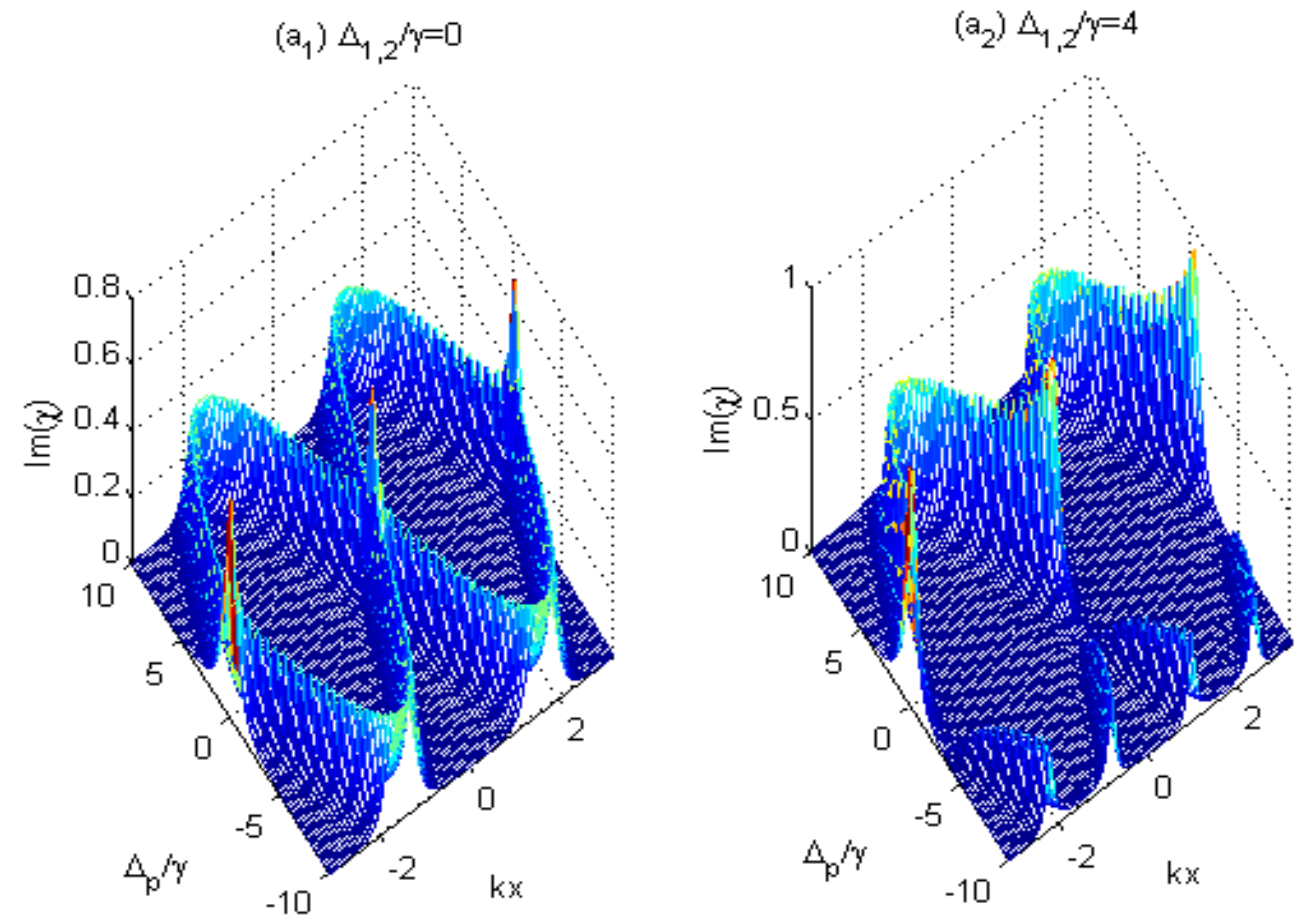

Figure 4. The 3D absorption spectra of $\operatorname{Im}(\mathrm{X})$ as a function of $k x(-\pi \leq k x \leq \pi)$ and $\Delta_{\mathrm{p}} / \mathrm{Y}$ for $\gamma_{1}=\gamma_{2}=\gamma_{p}=y, \Gamma_{i j}=\Gamma_{0}=3, \Gamma_{23}=\Gamma_{24}=0, \Omega_{1}=\gamma, \Omega_{2}=20 \gamma$, and different values of the detunings (a $\left.\mathrm{a}_{1}\right) \Delta_{1}=\Delta_{2}=0$ and $\left(\mathrm{a}_{2}\right) \Delta_{1}=\Delta_{2}=4 \mathrm{\gamma}$.

$\left(\Delta_{1}=\Delta_{2}=0\right)$, Figure $\left(4 a_{1}\right)$ show that the absorption spectra are symmetric with respect to $\Delta_{\mathrm{p}}=0, \mathrm{kX}=0$ and have a sharp peak at resonance. Thus, strong localization of the atom can occur in a narrow spectral region at the value of $\Delta_{\mathrm{p}}=0$, whereas the weak localization is possible only for a nonzero detuning $\Delta_{p}$. 

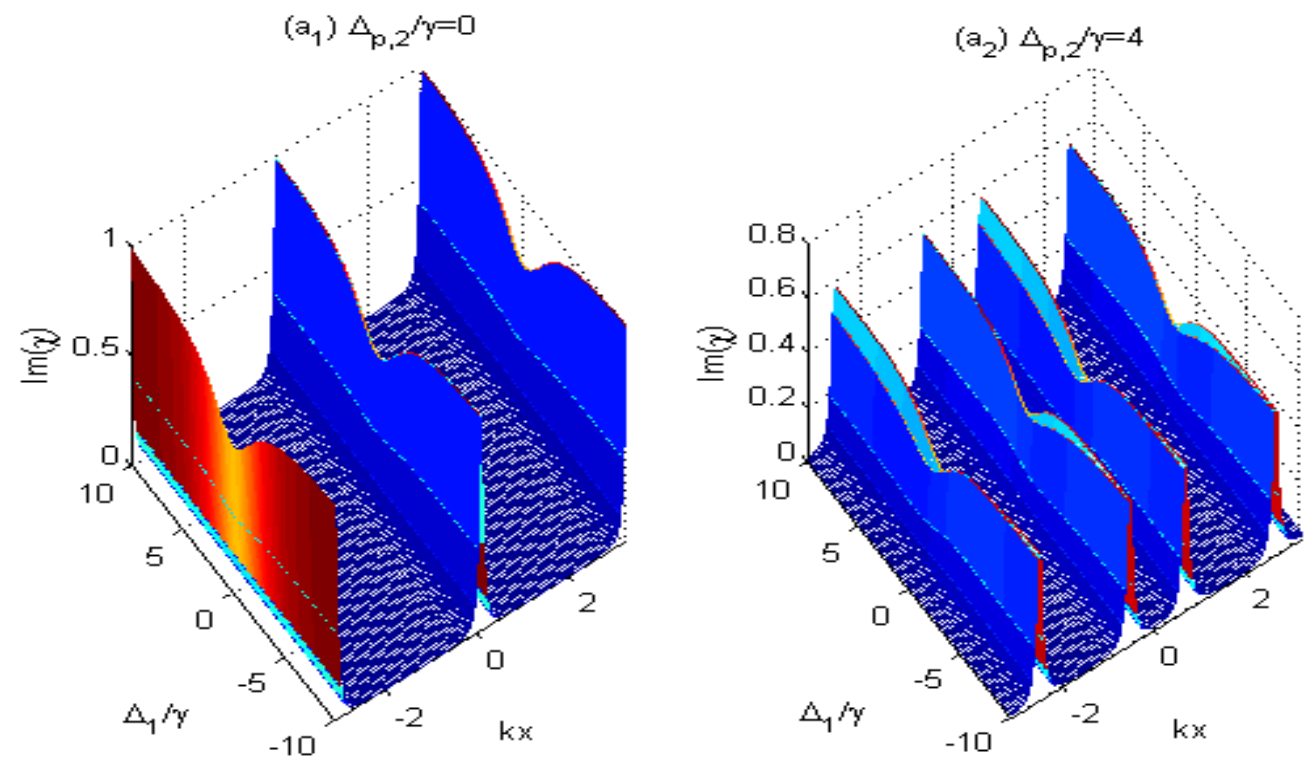

Figure 5. The 3D absorption spectra of $\operatorname{Im}(X)$ as a function of $k x(-\pi \leq k x \leq \pi)$ and $\Delta_{1} / Y$ for the same parameters as in Figure 4 but for different values of the detunings $\left(a_{1}\right) \Delta_{p}=\Delta_{2}=0, \quad\left(a_{2}\right)$ $\Delta_{\mathrm{p}}=\Delta_{2}=4 \mathrm{\gamma}$.
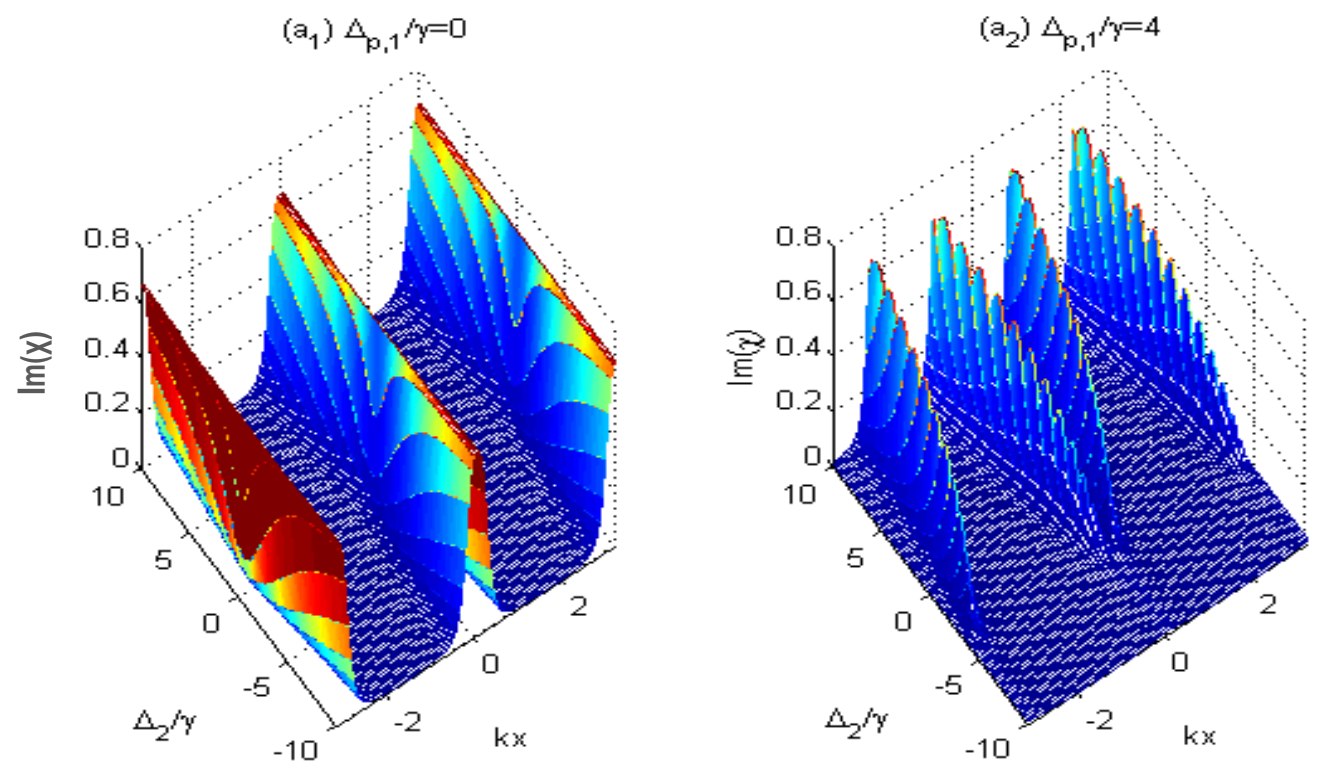

Figure 6. The 3D absorption spectra of $\operatorname{Im}(X)$ as a function of $k x(-\pi \leq k x \leq \pi)$ and $\Delta_{2} / y$ for the same parameters as in Figure 4 but for different values of the detunings $\left(a_{1}\right) \Delta_{p}=\Delta_{1}=0,\left(a_{2}\right)$ $\Delta_{\mathrm{p}}=\Delta_{1}=4 \mathrm{\gamma}$.

For off-resonance condition $\left(\Delta_{1}=\Delta_{2}=4\right)$, the behavior of $\operatorname{Im}(X)$ is changed qualitatively as well as quantitatively when the detuning $\Delta_{p}$ is varied from negative to positive values as presented in Figure $\left(4 a_{2}\right)$. Note that, "the super- localization" that is, the sharp peaks of the spectra are positioned near the nodes at $\mathrm{kx}=0, \pm \pi$ and at certain value of the detuning $\Delta_{p}(-10,0,4)$.

Figure 5 show the absorption spectra versus kx for various values of the detuning $\Delta_{1}$. We observe in Figure $\left(5_{a_{1}}\right)$ that, 
at the resonance detunings $\left(\Delta_{\mathrm{p}}=\Delta_{2}=0\right)$, the absorption spectra of $\operatorname{Im}(X)$ show the localization of the atom in a sub-wavelength regime with sharp peaks around the nodes of the standing-wave field and exhibit a small dip at $\Delta_{1}=0$. For off-resonance condition $\Delta_{p}=\Delta_{2}=4$, as shown in Figure $\left(5 \mathrm{a}_{2}\right)^{\prime \prime}$ the absorption spectra split into four peaks at the nodes of the standing-wave field with the same height for all values of the positions, with a uniform profile and have a small dip at $\Delta_{1}=0$. The subwavelength of the localization atom are observed.

In Figure $\left(6 \mathrm{a}_{1}\right)$ at $\left(\Delta_{1}=\Delta_{\mathrm{p}}=0\right)$, we obtain two equally probable peaks appear at the nodes of the standingwave field. The spectra are symmetric with respect to $k x=0$. It is seen that the spectral width increases with increasing $\left|\Delta_{2}\right|$. For off-resonance condition $\Delta_{1}=\Delta_{p}=4$, in Figure $\left(6 \mathrm{a}_{2}\right)$ the absorption spectra are symmetric with respect to $k x=0$ and a different sort of the spectra are observed in the positive range of the driving-detuning $\Delta_{2}$ and the atom localization is predicted for a certain values of $\Delta_{2}$, in a similar manner to that for a two level system ( $\mathrm{Xu}$ and $\mathrm{Hu}, 2007$ ). As indicated in Equation (19) the position of the peaks can be estimated approximately in the present situation $\left(\Delta_{\mathrm{p}}=\Delta_{1}=\Delta_{2}=0\right)$ by: $\mathrm{KX}= \pm \sin ^{-1}\left(\frac{\Omega_{1}}{\Omega_{2}}\right)+n \pi$.

We note that the position of the peaks are dependant on the value of the ratio $(R)$ of the Rabi frequencies of the control and standing-wave fields $\left(R=\frac{\mathbb{n}_{1}}{\mathbb{\Omega}_{z}}\right)$. Thus, for very small values of the ratio $R<<1$, the maximum peaks are located around the nodes of the standing-wave fields that is, at $k x \approx n \pi$. Meaning that, for adequate choice of the ratio $R$ results in increasing the zone for the atom localization.

\section{Conclusion}

We investigated a four-level atom $\mathrm{V}$-type atomic system interacting with the three fields (control, probe and pump) for achieving the localization of the atom with coherence dephasing rates taken into account. We found that the coherence dephasing rates and the detunings of the fields affected qualitatively as well as quantitatively on the atom localization. We can conclude the results as:

(1) When the dephasing rates $\Gamma_{23}=\Gamma_{24}=0$, we obtain a strong localization through sharply peak (four sharply peaks) at resonance detunings (off resonance detunings),

(2) At small dephasing rates $\left(\Gamma_{\mathrm{ij}}=0.1\right)$ for off resonance detunings, we can find strong sub-wavelength, (3) The strong localization of the atom can occur in a narrow spectral region at the value of detuning $\Delta_{p}=0$ when $\left(\Delta_{1}=\Delta_{2}=0\right)$,

(4) When $\left(\Delta_{1}=\Delta_{2}=4\right)$, the super- localization is achieved near the nodes at $k x=0, \pm \pi$ and at certain value of the detuning $\Delta_{p}(-10,0,4)$,

(5) The absorption spectra exhibited a dip at $\left(\Delta_{1}=0\right)$ for resonance and off Resonance conditions $\left(\Delta_{p}=\Delta_{2}=0\right.$ and $=4)$,

(6) The localization is predicted in the positive range of the driving detuning $\left(\Delta_{2}\right)$ when $\left(\Delta_{1}=\Delta_{p}=4\right)$.

\section{REFERENCES}

Affolderbach C, Knappe S, Wynands R (2002). Electromagnetically induced transparency and absorption in a standing wave. Phys. Rev. A. $65: 043810$.

Agarwal GS, Kapale KT (2006). Subwavelength atom localization via coherent population trapping. J. Phys. B: At. Mol. Opt. Phys. 39:3437.

Ding C, Li J, Zhan Z, Yang X (2011). Two-dimensional atom localization via spontaneous emission in a coherently driven five-level M-type atomic system. Phys. Rev. A 83:063834.

Faist J, Capasso F, Sirtori C, West KW, Pfeiffer LN (1997). Controlling the sign of quantum interference by tunneling from quantum wells. Nature 90:589.

Ghafoor F, Qamar S, Zubairy MS (2002). Atom localization via phase and amplitude control of the driving field. Phys. Rev. A 65:043819.

Jun X, Qian L, Wen-chao Y, Xiang-dong C, Xiang-ming H (2008). Subhalf-wavelength localization of a two-level atom via tri-chromatic phase Manipulation. Phys. Letts. A. 2:6032-6036.

Kien FL, Rempe G, Schleich WP, Zubairy MS (1997). Atom localization via Ramsey Inter-ferometry: A coherent cavity field provides a better resolution. Phys. Rev. A 56:2972.

Macovei M, Evers J, Keitel CH, Zubairy MS (2007). Localization of atomic ensembles via super fluorescence. Phys. Rev. A. 5:033801.

Meystre P, Sergent M (1999). Elements of Quantum Optics, $3^{\text {rd }}$ ed., springer-verlag, Berlin.

Osman KI (2005). The effect of spread of the atomic wave function on some statistical properties of the field. Int. J. Quantum Inf. 3:339-350.

Paspalakis E, Knight PL (2001). Localizing an atom via quantum interference. Phys. Rev. A. 63:065802.

Qamer S, Zhu SY, Zubairy MS (2000). Atom localization via resonance fluorescence. Phys. Rev. A. 61:063806.

Sahrai M, Tajalli H, Kapale KT, Zubairy MS (2005). Subwavelength atom localization via amplitude and phase control of the absorption spectrum. Phys. Rev. A 72:013820.

Scully MO, Zubairy MS (1997). Quantum Optics, Cambridge University Press. Cambridge.

Wang Z, Yu B, Zhu J, Cao Z, Zhen S, Wu X, Xu F (2012). Atom localization via controlled spontaneous emission in five-level atomic system. Ann. Phy. 327:1132-1145.

Xu J, Hu XM (2007). Localization of a two-level atom via absorbtion spectrum. Phys. Letts. A. 364:208-213. 